\title{
La interdisciplinariedad en la formación contable: estudio del currículo en el programa de Contaduría Pública de la Universidad Santo Tomás, Bogotá
}

\author{
Gabriel Enrique Moreno Sánchez ${ }^{1}$ \\ Óscar Yamith Duque Cruz ${ }^{2}$
}

Recibido: 17 de febrero de 2016

Aprobado: 19 de mayo de 2016

\begin{abstract}
Moreno, G. \& Duque, O. (2016). La interdisciplinariedad en la formación contable: estudio del currículo en el programa de Contaduría Pública de la Universidad Santo Tomás, Bogotá. Activos, 26, 25-60.
\end{abstract}

Clasificación JEL: B49, I 29

1 Psicólogo egresado de la Universidad INCCA de Colombia. Magíster en Calidad y Gestión Integral. Docente e investigador de la Facultad de Contaduría Pública de la Universidad Santo Tomás. Miembro del grupo de investigación Contaduría: Información, Control e Impacto Social y del Observatorio Colombiano de Educación Contable OCEC. Correo electrónico: gabrielmoreno@usantotomas.edu.co

2 Administrador de empresas egresado de la Universidad Antonio Nariño. Magíster en Educación de la Universidad Santo Tomás. Docente e investigador de la Facultad de Contaduría Pública de la Universidad Santo Tomás. Miembro del grupo de investigación Contaduría: Información, Control e Impacto Social y del Observatorio Colombiano de Educación Contable OCEC. Correo electrónico: oscarduque@usantotomas.edu.co 


\section{Resumen}

El presente trabajo refleja una de las preocupaciones fundamentales en el quehacer de la enseñanza de la contaduría pública en la educación superior: la interdisciplinariedad y transdisciplinariedad que se suelen argumentar como criterios en la construcción de los procesos educativos, ¿̇se evidencian en todos los procesos formativos o solamente son variables que se expresan de manera desarticulada en los currículos?

La intención del presente trabajo es revelar la importancia de los conceptos interdisciplinariedad y transdisciplinariedad en los procesos de formación universitaria, particularmente para el caso del programa de Contaduría Pública de la Universidad Santo Tomás. En el desarrollo del trabajo se concluye que, aunque los conceptos señalados tienen un alto nivel de relevancia, no aparecen claramente desarrollados en el despliegue formativo que le sigue al currículo y tienen problemas de articulación y coherencia.

\section{Palabras claves}

Currículo, interdisciplinariedad, transdisciplinariedad, formación contable.

Moreno, G. \& Duque, O. (2016) Interdisciplinarity in Accounting Training: Analysis of Curriculum of Accounting program of Santo Tomas University, Bogotá. Activos, 26, 25-60.

\section{Abstract}

The present work reflects one of the fundamental concerns in the task of teaching accounting in higher education: the interdisciplinarity and transdisciplinarity that are usually argued as a criterion in the construction of educational processes is evident in all, or are they only variables that are expressed in a disjointed way in the curricula?

The intention of the present work is to reveal the importance of interdisciplinary and transdisciplinarity concepts in the university formation processes, 
and particularly for the case of the Accounting formation program at the Santo Tomás University, in the development of the work it is concluded that although the concepts indicated Have a high level of relevance, are not clearly developed in the formative deployment that follows the curriculum, and have problems of articulation and coherence.

\section{Keywords}

Curriculum, interdisciplinarity, transdisciplinarity, accounting education.

\section{Moreno, G. \& Duque, O. (2016). Interdisciplinarité dans l'éducation comptable: révision du programme d'études en comptabilité pu- blique de l'Université Santo Tomás, Bogota. Activos, 26, 25-60.}

\section{Résumé}

Ce travail reflète l'une des préoccupations fondamentales du travail de l'enseignement de la comptabilité publique dans l'enseignement supérieur: l'interdisciplinarité et la transdisciplinarité qui se font souvent valoir comme des critères dans la construction des processus éducatifs. Sont-ils évidents dans tous les processus éducatifs ou sont-ils seulement des variables qui s'expriment de façon décousue dans les programmes éducatifs?

Lobjectif de ce travail est de révéler l'importance des concepts interdisciplinaires et transdisciplinaires dans le processus de l'enseignement universitaire, en particulier dans le cas du programme de comptabilité publique de l'Université Santo Tomás. Á travers le développement de ce travail on conclut que, même si les éléments mentionnés ci-dessus ont un haut niveau de pertinence, ils ne sont pas clairement développés dans le déploiement de la formation que suit le programme d'études et qu'ils ont des problèmes d’articulation et de cohérence.

\section{Mots-clés}

Programme, interdisciplinarité, transdisciplinarité, formation comptable. 


\section{Introducción}

La interdisciplinariedad es entendida como la posibilidad de relación entre varias disciplinas o ramas del conocimiento que enriquecen la perspectiva del escenario, creando posibilidades y abriendo horizontes más amplios a la realidad que se quiere dar a conocer; esta permite reducir el impacto de la especialización y la fragmentación del conocimiento. Carvalho et al. (2006) señalan que los hechos, las situaciones, los fenómenos no escogen campos específicos para aparecer, sino que son producto de relaciones concretas entre diversos elementos y, en este sentido, su comprensión implica el concurso de más de una manifestación disciplinaria.

Con este concepto en mente, el presente trabajo revisa el Plan de Estudios de Contaduría Pública (PECP) de la Universidad Santo Tomás sede Bogotá, para identificar los criterios de interdisciplinariedad y transdiciplinariedad. Esta será como la fase inicial de estudios, que deberá conducir a un análisis comparativo con otros programas de Contaduría Pública en términos de los criterios ya reseñados.

El documento propone una metodología cualitativa en el análisis y utiliza un cuestionario para el levantamiento de la información, así como una entrevista semiestructurada. Los resultados de estos instrumentos son contrastados con los conceptos teóricos y con los documentos curriculares de la Institución y del programa, con el fin de establecer si las intencionalidades de la interdisciplinariedad y de la transdisciplinariedad son identificadas por los estudiantes y los docentes del programa señalado.

\section{Planteamiento del problema}

El currículo es la forma como se materializa el proceso educativo, en él se formula el camino que se va a recorrer en términos de la formación profesional y académica, y revela todas las intencionalidades del proceso educativo, incluyendo la concepción que la institución educativa tiene con referencia al ser humano (aspecto antropológico), la sociedad (aspecto 
sociológico) y a las estrategias derivadas del respectivo pensamiento institucional (aspectos filosófico y pedagógico).

En este orden de ideas, aspectos como la cultura, el ambiente social, económico, político y el desarrollo de la ciencia serán orientadores del diseño y de implementación curricular, lo cual es válido tanto para las instituciones académicas como para todos y cada uno de los programas que las componen.

En este sentido, hay que señalar que el concepto currículo integra filosófica, conceptual e instrumentalmente los aspectos propios de la formación (universitaria en este caso), asignando una mayor relevancia a unos aspectos fundamentales que son los que, a la larga, terminan expresando el sello institucional y del programa. Como puede deducirse, el currículo es mucho más que un documento que expresa un mundo ideal para la formación de los nuevos profesionales, es también una expresión práctica de dichas ideas, las cuales se ven reflejadas en los documentos institucionales.

Es frecuente que al analizar los currículos de cualquier programa de formación se encuentre una muy buena articulación entre sus diferentes componentes, sin embargo, al ser la expresión concreta de los fines del proceso educativo, el currículo está en permanente proceso de evolución y cambio, por ende, en muchas ocasiones hay una divergencia entre los discursos oficiales y las prácticas cotidianas.

En términos más puntuales, en su documento de Política Curricular, la Universidad Santo Tomás plantea formalmente la interdisciplinariedad "como aquella característica que reconoce las diferencias y complementariedades entre las disciplinas y privilegia el trabajo mancomunado y la transdisciplinariedad”, así como la “(...) transdisciplinariedad garantiza una visión de totalidad, por la construcción de discursos que trascienden las disciplinas mismas..." (Universidad Santo Tomás, 2004 (a), p. 23). Estos conceptos son válidos para todas las disciplinas académicas y permiten plantear la pregunta sobre en qué sentido y dimensión la contaduría pública, tratándose de un campo que por definición interactúa con otras disciplinas, cumple con dichos criterios. 
Ello conduce a plantear la pregunta central del presente trabajo: ¿es posible evidenciar la interdisciplinariedad en el currículo del programa de Contaduría Pública de la Universidad Santo Tomás sede Bogotá?

Con esta pregunta en mente, el presente trabajo busca identificar la presencia de los criterios y de las prácticas de interdisciplinariedad en el currículo del programa de Contaduría Pública de la Universidad Santo Tomás Bogotá.

Para ello, es necesario evidenciar si la interdisciplinariedad se hace explícita en los propósitos de formación del programa de Contaduría Pública de la Universidad Santo Tomás sede Bogotá, a la vez que se hace necesario identificar la coherencia que existe entre la formulación explícita del programa de Contaduría Pública y las prácticas concretas de interdisciplinariedad a lo largo del proceso de la formación.

\section{Aproximación conceptual}

\section{Definición de currículo}

El curriculum no es solamente el compendio de contenidos de un programa de formación académica, es la fórmula de contenidos la cual se debe suministrar a un profesional en formación para que, al cumplir su proceso educativo, tenga las características deseadas para la función profesional que va a desempeñar. Así pues, para una misma profesión se pueden adquirir diferentes perfiles de desempeño, con el establecimiento de unos énfasis diferentes en los contenidos y en su formación ética (Agray Vargas, 2010). La delimitación del campo del currículo ha dependido de las concepciones del término, las cuales se han visto influenciadas por la importancia de la escuela analítica de filosofía y su impacto sobre los estándares educativos. Con el fin de evitar la tentación de una visión restringida del concepto, se ha optado por limitar su definición a las experiencias diseñadas para los estudiantes, incluyendo aspectos más globales de la situación escolar, y limitarse a una breve conceptualización de los planes de estudio. 
En términos de las definiciones institucionales de la Universidad Santo Tomás, "la palabra currículo viene del latín curriculum (derivado del verbo currere $=$ correr). Ya en Cicerón denotaba carrera, espacio donde se corre, espacio tiempo, modo de vivir" (Universidad Santo Tomás, 2004 (b), p. 19).

En efecto, Cicerón usó el concepto para referirse al transcurrir de la vida y del tiempo: "(...) curriculum temporis, curriculum annorum, curriculum vivendi, curriculum vitae ..." (Universidad Santo Tomás, 2004 (b), p. 19). En el siglo XIX, se usaba en español la expresión latina "carrera de la vita" o "currículum vivendi", lo cual traduce la idea de que la educación formal es, en realidad, un camino.

En el currículo se debe ubicar al educando en el entorno en que desarrollará sus actividades y generar en él un sentido de pertenencia. Se deberá establecer que los contenidos académicos y la comprensión de ellos sean pertinentes al tipo de profesional que se va a formar; el currículo es un instrumento potente que permite la transformación de la enseñanza, en el que se hace públicamente disponible la experiencia, consistente en intentar poner en práctica una propuesta educativa (Stenhouse, 2003).

Autores como Kemmis ((1998), citado en Duque y Pinzón, 2014) indican tres teorías sobre currículo:

- Teoría técnica sobre el curriculum: El curriculum es un contexto caracterizado por necesidades y objetivos sociales que conllevan el desarrollo de programas para así alcanzar objetivos de la sociedad.

- Teoría práctica del curriculum: La sociedad y la cultura son vistas como un sustrato y por ello busca el desarrollo de personas educadas que sean capaces de pensar de manera crítica.

- Teoría crítica del curriculum: Las estructuras sociales no son racionales como comúnmente se piensa, sino que dichas estructuras sociales son creadas por procesos y prácticas que se han deformado de la realidad en la que se encuentra el sujeto. 
Por su parte, Bolívar (2008) ilustra las categorías bipolares de currículo:

- Currículo como curso de estudios versus curso de vida

- Como contenidos planificados versus experiencias vividas

- Como producto (documento) versus proceso contextualizado

- Como intención versus realidad.

Todo lo anterior permite divisar que el currículo ha pasado por distintas etapas de conceptualización y, por consiguiente, por la lupa de diferentes focos teóricos, lo cual dificulta establecer conceptos homogéneos para su estudio.

La disciplina del currículo se desarrolló inicialmente como una asociación directa al concepto y a la perspectiva de los programas y planes de estudio. Los estudios sobre educación y, por lo tanto, de currículo, provinieron originalmente de Europa (desde principios de la década de los sesenta), pero con el tiempo comenzaron a llegar autores de escuelas norteamericanas en las que el Estado tenía poca participación en el control de los procedimientos que desarrollan el currículo (programas, planes, malla curricular), hasta que con la finalización de la Segunda Guerra Mundial se implementaron reformas escolares que fundamentaron una interesante participación de varios agentes de interés en la educación.

En los años setenta, el tema fundamental de los estudios curriculares buscaba "centrarse en lo local, por ejemplo, dio la atención al currículum oculto (...) y la aparición de tradiciones etnográficas y la investigación sobre el aula en los EE. UU." (Popkewitz, 2007, p. 2). Esto implicó un énfasis sobre la selección, organización y evaluación de lo que se definió en ese momento como el "conocimiento legítimo de la escuela".

En América Latina, a su vez, fueron desarrollándose nociones sobre el currículo: "En México, por ejemplo, hubo aportaciones a conceptos como 'diagnóstico de necesidades' (Taba, 1974) o 'fuentes y filtros' para el diseño curricular al establecer conceptos como marco de referencia de un plan de estudios, análisis de la práctica profesional..." (Díaz, 2003). Así mismo, 
Zabalza (1801 citado por Díaz, 2003, p. 6) propuso el desarrollo curricular como concepto enfrentado al de diseño curricular, al criticar que el currículo no podía comprenderse como si se tratase únicamente de una estructura.

Díaz (2003) expresó que, en este sentido, la perspectiva de los planes y programas aumentó. Se debían atender, por un lado, las necesidades institucionales del sistema educativo, a la vez que era necesario seleccionar los contenidos y la formación como un tema social y no meramente institucional o particular. Este esquema generaría a posteriori un cambio radical en las condiciones de práctica docente, ya que ahora se deberían conocer y dominar los contenidos institucionalizados y la didáctica propuesta por expertos, restringiendo la autonomía de la práctica docente.

Entonces, desde este enfoque, el currículo debe enfocarse en los lineamientos legales y normativos del país donde se desarrolla. Por ello "el análisis de educación contable no se puede desligar de la educación universitaria en el país" (Gracia, 2002), lo cual significa que debe ligarse la educación contable de las instituciones, a la educación contable de la nación.

En este sentido, Martínez (2002) señala:

Las reformas curriculares de los programas contables en Colombia han tenido una vida demasiado precaria y más han obedecido a una respuesta coyuntural y pragmática para atemperar los programas a las necesidades del entorno empresarial dominante y a las exigencias dogmático-normativas de los cambios de regulación contable internacional”. (Martínez, 2002, p. 119).

Según Ortiz (2006), el currículo se puede ver fundamentalmente de dos formas: una llamada tradicional, que lo aborda desde el conocimiento fragmentado y asociado con procesos psicológicos como la memorización, que incluye una actuación docente desde una sincronizada programación de los contenidos y tiempos dedicados al estudio de dichos contenidos. La otra forma propone una visión integrada centrada en el estudiante, con un rol diferente del docente, el cual actúa como tutor, con una integración entre disciplinas propia del modelo construccionista. 
Se percibe entonces una clara discontinuidad entre los elementos de la teoría pedagógica, la cual se expresa en la reproducción de una actividad educativa en la que la asimilación de la disciplina se reduce a los aspectos más formales de la personalidad y de la vida. En las definiciones anteriores se evidencia que lo curricular incluye variados aspectos, los cuales se concretan en el plan de estudios y en algunas estrategias pedagógicas y didácticas, lo que posteriormente tiene impacto directo en la comunidad académica, la calidad y la proyección y cubrimiento de las necesidades sociales a las que se debe ver abocado el contador público en la actualidad. Un análisis comparativo de dichos enfoques puede identificarse en la tabla 1 .

Tabla 1. Tipos de currículo

\begin{tabular}{l|l}
\hline \multicolumn{1}{c|}{ Tradicional } & \multicolumn{1}{c}{ Integrado } \\
\hline $\begin{array}{l}\text { Las asignaturas son espacios territo-- } \\
\text { riales para propósitos del maestro. } \\
\text { Son categorías abstractas. }\end{array}$ & $\begin{array}{l}\text { Las asignaturas integradas son espacios en } \\
\text { los que se comparten destrezas, conceptos y } \\
\text { actitudes interdisciplinariamente; se ofrece el } \\
\text { conocimiento de manera concreta. }\end{array}$ \\
\hline $\begin{array}{l}\text { El conocimiento, las destrezas y los } \\
\text { conceptos están fragmentados y } \\
\text { separados. }\end{array}$ & $\begin{array}{l}\text { El conocimiento, las destrezas y los conceptos } \\
\text { se ofrecen haciendo conexiones con las ideas } \\
\text { nuevas, los cursos y los escenarios fuera de la } \\
\text { escuela. Se busca su integración. }\end{array}$ \\
\hline $\begin{array}{l}\text { El estudiante es visto como alguien } \\
\text { importante. Sin embargo, se le ofrece } \\
\text { la enseñanza de forma igual para } \\
\text { todos. }\end{array}$ & $\begin{array}{l}\text { El estudiante es importante, pero aprende a } \\
\text { su propio ritmo porque la enseñanza debe ser } \\
\text { individualizada. }\end{array}$ \\
\hline $\begin{array}{l}\text { El estudiante recibe información que } \\
\text { muchas veces no tiene significado } \\
\text { para sí. }\end{array}$ & $\begin{array}{l}\text { El estudiante utiliza la información de su entor- } \\
\text { no para adquirir aprendizaje genuino. }\end{array}$ \\
\hline $\begin{array}{l}\text { El estudiante memoriza, interpreta y } \\
\text { comprende la información dada, que } \\
\text { muchas veces no se relaciona con su } \\
\text { situación particular. Oye más y hace } \\
\text { menos. }\end{array}$ & $\begin{array}{l}\text { El estudiante interpreta, generaliza, aplica, } \\
\text { hace analogías y ejemplariza, partiendo de sus } \\
\text { experiencias y de problemas que pueden ser } \\
\text { personales o de su ambiente. Oye menos, pero } \\
\text { hace más. }\end{array}$ \\
\hline $\begin{array}{l}\text { El maestro es poseedor del conoci- } \\
\text { miento y lo vacía en sus estudiantes } \\
\text { como si estos fueran meras vasijas } \\
\text { para recibirlo. }\end{array}$ & $\begin{array}{l}\text { El maestro es un facilitador que ayuda a que los } \\
\text { estudiantes lleven a cabo procesos del pen- } \\
\text { samiento que le hagan entender y asimilar la } \\
\text { información con significado. }\end{array}$ \\
\hline
\end{tabular}




\begin{tabular}{l|l}
\hline $\begin{array}{l}\text { El maestro presenta hechos y } \\
\text { destrezas de unidades programadas } \\
\text { aisladamente. }\end{array}$ & $\begin{array}{l}\text { El maestro presenta hechos y destrezas por me- } \\
\text { dio de temas generadores o metaconceptos. }\end{array}$ \\
\hline $\begin{array}{l}\text { La enseñanza se ofrece mediante te- } \\
\text { mas abstractos y artificiales que han } \\
\text { sido impuestos por los adultos. }\end{array}$ & $\begin{array}{l}\text { La enseñanza se ofrece mediante temas ricos } \\
\text { y provocativos que surgen de la vida diaria del } \\
\text { estudiante y que son reales. }\end{array}$ \\
\hline $\begin{array}{l}\text { Se espera que el estudiante posea } \\
\text { las destrezas y los conceptos que le } \\
\text { permitan funcionar efectivamente } \\
\text { en la sociedad del futuro. }\end{array}$ & $\begin{array}{l}\text { Se espera que el estudiante posea las destrezas y } \\
\text { los conceptos que le permitan funcionar efecti- } \\
\text { vamente en la sociedad del futuro. }\end{array}$ \\
\hline
\end{tabular}

Fuente: Construcción propia a partir de Ortiz (2006).

En conclusión, el currículo se acepta como un plan de acción que se crea para la solución de problemas de la profesión y su contexto. Con él se organiza, dirige, ejecuta y controla el proceso en la formación de profesionales, con la participación de los actores interdisciplinarios de la comunidad académica; tiene como requisitos el ser flexible, comprensible y adaptable, con el fin de posibilitar un abordaje integrador para una realidad que por definición es integrada.

\section{Los usos disciplinarios}

\section{Interdisciplinariedad}

Históricamente, el estudio de los diferentes fenómenos del mundo ha estado asociado con su reducción y simplificación, que permitan que sea abordable o por lo menos más fácilmente analizable. Sin embargo, es sabido que la segmentación es un artilugio mental, útil en algunos casos, pero nada más, tal como lo plantea Morin (2001, p. 2):

... la tradición del pensamiento que forma el espíritu de las escuelas elementales ordena a reducir lo complejo a lo simple, es decir, separar lo que está ligado, unificar lo que es múltiple y eliminar todo lo que trae desórdenes o contradicciones a nuestro entendimiento. 
Esta visión indudablemente facilita la revisión y el análisis, sin embargo, no hace lo mismo con la comprensión. Es esto lo que se conoce como el enfoque disciplinario, el cual tiene como elemento de soporte la fortaleza conceptual en un aspecto pequeño y compartimentalizado de un fenómeno o problema, lo cual de alguna forma se explica en la gran cantidad de conocimientos que la humanidad ha ido acumulando a través del tiempo; no obstante, los enfoques excesivamente disciplinarios terminan en un reduccionismo metodológico que permite explicar, mas no necesariamente comprender.

Es de anotar que la visión compartimentalizada ya mencionada se inscribe perfectamente en la visión de la Modernidad, la filosofía cartesiana y los elementos que le dieron vida a lo que se conoce como la racionalidad del mundo moderno, en particular, la racionalidad instrumental, donde cada tema o aspecto debe encajar a la perfección y estar orientado a la solución de un problema, en algunos casos confundiendo el concepto de disciplina con el de profesión. Esta forma de estudio y análisis se basa en lo simple y en la eliminación del contexto en el que opera un problema, en el cual termina eliminándose, sobrevalorándose lo parcial y fragmentario, en detrimento de la comprensión global.

Este modelo simplificador dista de ser deseable en la medida en que cuando se pretende hablar de disciplina científica debe entenderse esta como la que proporciona y sustenta un conjunto armonizado de conocimientos sobre un fenómeno en sólidas bases conceptuales, epistemológicas e investigativas de un campo del saber. Nótese que, en todo caso, el término disciplina parte de reconocer que el entorno es complejo, que no es único y que cualquiera que fuere el tema por abordar, la disciplina reconoce sus propios límites, por ello habla de campos de saber, dando a entender de entrada que la realidad es global e interactuante.

En términos de Castro (2013, p. 35):

... para lograr conocimiento válido, el objeto de conocimiento solicita dividirse en partes cada vez más pequeñas. No existe ciencia de la totalidad, solamente ciencia de parcelas de la realidad. No puede haber conocimiento 
del todo, sino solo de las partes. Esto significa que los saberes holísticos no configuran conocimientos que pertenezcan al ámbito de la ciencia.

Este es el paradigma cartesiano en su máxima expresión. Adicionalmente, hay que contemplar las paradojas que pueden presentarse cuando se trabaja desde el concepto de lo disciplinario; este pareciera favorecer el aprendizaje memorístico en la medida en que su énfasis se encuentra en los aspectos teórico-conceptuales. Por el contrario, desde la visión interdisciplinaria, el énfasis se encuentra en los aspectos relacionales de los cuerpos conceptuales pertenecientes a varias disciplinas. Aquí se formula una primera paradoja fundamental: el ser común en los programas de formación identificar la pretensión de formar simultáneamente desde lo disciplinario, pero abandonando las prácticas memorísticas.

La anterior es una forma de sustentar, en términos de argumentos psicológicos, por qué a pesar de la intención verbalizada de acabar con la memorística, ello no es tan fácil mientras se continúe con visión fundamentalmente disciplinaria. Es así como, según Torres (2006, p. 116):

... la psicología de la Gestalt como, posteriormente, la psicología cognitiva y social llegan continuamente a la conclusión de la necesidad de una mayor significatividad de los contenidos que la institución escolar debe manejar. Obviamente, una presentación fragmentada no va a cubrir tal requisito.

Ahora bien, si se habla de interdisciplinariedad, se estaría mencionando de entrada un tema de mayor envergadura e inscrito en un nivel superior al de la disciplinariedad, del cual es una respuesta en la medida en que, aunque la disciplina no fue concebida como reduccionista, terminó allí, tal vez por la ya mencionada acumulación histórica del conocimiento. Debe mencionarse también que el concepto de interdisciplinariedad nada tiene que ver con una forma de sincretismo científico, es decir, como una especie de amalgamiento que puede llegar a ser inconveniente en la medida en que se entienda como una mera fusión de saberes.

En este orden de ideas, surgen las siguientes preguntas: ¿cómo se explica la interdisciplinariedad?, ¿acaso como un elemento derivado de 
la disciplinariedad? Para apoyar una respuesta en este sentido, Miñana (2000) afirma:

Cuando una disciplina específica aborda un problema cuya comprensión la supera o cuando [en el] interior de ella misma se cuestionan sus teorías o sus fronteras, es cuando puede aparecer la interdisciplinariedad. (Miñana, 2000, p. 10).

Tal vez por ello se intenta explicar la interdisciplinariedad como sinónimo de holística. Sin embargo, debe entenderse que no existe la interdisciplinariedad sin la presencia previa de la disciplinariedad. Es por esta razón que Tamayo y Tamayo (2011) afirma que no es posible lograr una comprensión de lo interdisciplinario sin tener conocimiento previo de lo disciplinario.

Igualmente, debe comprenderse que la interdisciplinariedad tiene diferentes niveles de manifestación, no es una categoría única. Es así como Miñana (2000) habla de diferentes enfoques de interdisciplinariedad:

... uno de tipo relacional -interdisciplinariedad débil- (...) donde se piensa la interdisciplinariedad como "puente" que permite conectar dos o más disciplinas, moverse entre una y otra con distintos fines y de formas muy diversas; otro de tipo ampliativo, donde se crearían nuevos espacios y nuevas disciplinas con los aportes de varias -interdisciplinariedad fuerte-; finalmente, la aproximación radical, crítica, antidisciplinaria, que hace tabula rasa o que pretende situarse por fuera de las otras aproximaciones como podrían ser interpretadas las propuestas de Morin, o las críticas deconstruccionistas o [posdisciplinarias]... (Miñana, 2000, p. 12).

Esta aclaración de términos es importante debido a que tradicionalmente se asume como si la interdisciplinariedad fuese un concepto único e inequívoco. Esto implica que, para mencionarlo en relación con algún otro concepto, debe precisarse de qué nivel de interdisciplinariedad se está hablando, con el riesgo que conlleva hacer absolutos los términos, lo cual denota, a su vez, un segundo momento de inconsistencia. 
De esta forma, según lo formula Grajales (2011, p. 19):

En la actualidad, la interdisciplinariedad se traduce en la necesidad de concebir la realidad de forma holística y ya no comprendida únicamente desde áreas especializadas de conocimiento.

Podría afirmarse, en consecuencia, que la necesidad del abordaje interdisciplinario es un derivado lógico de la realidad misma, que así lo exige, por lo cual es necesario asumir si realmente se quieren comprender las dimensiones del término.

\section{Polidisciplinariedad}

Desde la Edad Media, los intereses que primaban en la sociedad de la época definieron las especialidades que las universidades terminaron por impartir. Así pues, las iniciantes instituciones de educación centraron su objetivo en la formación de eruditos en Derecho, Teología y en Ciencias Naturales.

Posteriormente surgieron las facultades, las cuales tenían como objetivo enseñar en distintas ramas de su saber, y las cuales se dividieron en dos vertientes: las facultades menores, donde se enseñaba Música, Gramática, Lógica y Matemáticas; y las facultades mayores, que impartían conocimientos en Derecho Civil y Canónico, Medicina y Teología.

El prefijo poli indica cantidad, indica muchos, es decir, que se refiere a una variedad de disciplinas que no suponen -en principio- una interrelación de estas para poder entender los fenómenos, son el fruto del interés por satisfacer las necesidades de la sociedad medieval, que buscaba ante todo el entendimiento de una realidad en particular.

Sin embargo, las transformaciones sociales de la Modernidad y la Posmodernidad guiaron a las universidades y sus facultades hacia otro rumbo, lo que ha conllevado que los objetivos y propósitos de formación también muten. Lo complejo de la sociedad y la interrelación de los subsistemas han 
generado una participación de los integrantes de la comunidad académica y la integración alternativa con otras disciplinas.

Morin (2001) manifiesta que existen ciencias multidimensionales, como la geografía, que cubre un extenso terreno partiendo de la geología y aterriza en el análisis de los fenómenos sociales y económicos; a otras las define como poliscópicas, las cuales abarcan la multidimensionalidad de la realidad humana hasta los aspectos más complejos de la humanización en la prehistoria. En este mismo sentido, el saber también ha optado por el agrupamiento de disciplinas que estaban en dispersión -la ecología científica y la cosmología son ejemplos claros de ciencias polidisciplinarias- que buscan no solo el estudio de un sector o un fragmento, sino más bien el análisis de un sistema complejo. Para Morin, entonces, lo polidisciplinario es la asociación de las disciplinas en torno de un proyecto o un objetivo que les es común.

Este enfoque invita entonces a la comunidad universitaria a la real integración de departamentos y a la "reintegración polidisciplinaria" (Morin, 2001, p. 5) en torno a un núcleo sistémico común en cualquier ámbito en el interior de la institución.

En este orden de ideas, la diferencia de lo polidisciplinario respecto a lo interdisciplinario estaría básicamente en la naturaleza temporal de aquella, sin que implique necesariamente una concepción integradora a lo largo de una línea de tiempo.

\section{Transdisciplinariedad}

Una gran variedad de sucesos hace progresar las ciencias y rompe con la lejanía de las disciplinas tradicionalmente distanciadas, bien sea por la diferenciación de conceptos, los aspectos cognitivos o la intromisión de una ciencia con las demás, a causa de las complejidades de algunas de ellas. La integración y la interdependencia de los saberes en algunos casos permiten articular los dominios disciplinarios en un sistema teórico que sea común. Las disciplinas se justifican desde lo particular y lo intelectual, siempre y 
cuando posean una visión que reconozca y permita la posibilidad de relacionarse y ser solidario con otras ciencias. La expansión en la actualidad de las disciplinas, sean estas académicas o no, acarrea un alto crecimiento de los saberes, y, en este sentido, cualquier intento de disminuir al ser humano a una simple definición y de discriminarlo en estructuras formales es discordante con el enfoque transdisciplinario.

Así pues, Ander-Egg (1994, citado en Miñana, 2000) indica que el sentido de la transdisciplinariedad no sugiere solamente el cruzamiento y la penetración de distintas disciplinas, más bien tiene como objetivo eliminar los límites existentes entre ellas y las integra en un solo sistema. La transdisciplinariedad no busca dominar un sinnúmero de disciplinas, sino, más bien, la posibilidad de permitir el ingreso de otras disciplinas que atraviesan o transitan dentro de ella.

Es así como Morin (1999-2000 citado en en Miñana, 2000, p. 16) sugiere que la transdisciplinariedad es un conjunto de "esquemas cognitivos que atraviesan las disciplinas y que a veces pueden ponerlas en trance", lo anterior entendido como que es aquella disciplina que asume su papel transversal o diagonal sobre las demás que conforman un mismo sistema. Por eso, se puede entender que la transdisciplinariedad reconoce la presencia de variados niveles de realidad, saberes y pensamientos, y hace emerger de estas confrontaciones nuevos datos que se articulan entre sí y generan nuevas visiones de dicha realidad, de dicha disciplina en torno a la cooperación, la articulación y el desarrollo de un proyecto común (Morin, 2010).

Como lo indica Martín-Barbero (2005), el punto de partida para entender y reconocer la transdisciplinariedad está en afirmar que no corresponde a una antítesis de las disciplinas, sino a un aspecto complementario de ellas, como aporte a los avances y a la gama de disciplinas que han surgido en los últimos tres siglos.

La transdisciplinariedad no significa descentralizar una disciplina, sino abrirla y extenderla, evitando el error de creer que es pura en sí y que no requiere de otros aportes no provenientes solamente de su conjunto de 
ciencias inmediatas o relacionadas, sino también de las ciencias humanas, culturales o de experiencias asociadas entre ellas. La transdisciplinariedad se fundamenta en la necesidad de salirse de la zona disciplinaria hacia un saber preparado para la multidimensionalidad que tiene la sociedad. Nicolescu (1996 citado por Roldán, 2008) indica, de esta misma manera, que la transdisciplinariedad concierne a lo que de manera simultánea está entre las disciplinas, por medio de distintas disciplinas y allende de toda disciplina, comprendiendo el mundo presente y buscando la unidad del conocimiento.

En este orden de ideas:

La transdisciplinariedad no es un diálogo ni tampoco un intercambio entre dos o más disciplinas, sino un devenir permanente de problemas que cambian todo el tiempo y que obligan a una renovación constante de la mirada. (Castro, 2013, p. 41).

En consecuencia, tal vez pueda pensarse en distintos niveles de lo asociado con lo disciplinario, como es lo disciplinario mismo, lo interdisciplinario y lo transdisciplinario, en una relación que significa un aumento del nivel de conocimiento de un fenómeno, que podría expresarse en la relación descripción-explicación-comprensión.

\section{Multidisciplinariedad}

Es un conjunto de disciplinas que se proponen simultáneamente, pero sin hacer aparecer explícitamente las relaciones entre ellas; es un sistema de un solo nivel y con objetivos múltiples que supone una relación de nocooperación (Organización para la Cooperación y Desarrollo EconómicoOCDE, citado en Miñana, 2000), lo cual permite tener diferentes ópticas disciplinarias válidas, por supuesto, pero sin diálogo entre disciplinas y, por lo tanto, el nivel de comprensión no aumenta.

La multidisciplinariedad se refiere a cómo un mismo problema o tema puede ser abordado desde diversas disciplinas sin que exista integración 
entre ellas, bien sea desde lo conceptual o desde lo metodológico. En este enfoque, cada una de las disciplinas asume su propia óptica y desde allí estudia; lógicamente ello no genera interdisciplinariedad, dado que cada una de las disciplinas abordantes asume el estudio desde su propio cuerpo epistemológico.

Es por lo anterior que la transdisciplinariedad, según lo planteado por Menéndez (1998), implica que al

... abordar cualquier campo determinado de la realidad caben múltiples disciplinas que confluyen en su resolución. La multidisciplinariedad da cuenta de las disciplinas, ciencias o ramas del conocimiento que tienen que ver y dan razón del saber sobre lo concreto de un problema. (Menéndez, 1998, p. 145).

Es decir, desde lo multidisciplinario no existe compromiso por el estudio relacionado ni mucho menos integrado. Puede observarse de esta forma que las que sí existen son diferentes ópticas, pero con inexistencia de diálogo entre las disciplinas; de esta forma, desde lo funcional, lo que hay son múltiples explicaciones a un hecho o fenómeno que al final es único e indivisible, lo cual puede considerarse un craso error.

Hay que tener presente también que estos enfoques diferenciales de un mismo fenómeno no son producto de una abstracción, la cual, desde lo metodológico, haría una aproximación diferente al problema, porque por lo menos sería explicitable la separación artificial del abordaje del problema.

\section{Pluridisciplinariedad}

Tamayo y Tamayo (2011) indica que es un conjunto de disciplinas cercanas y con gran afinidad en yuxtaposición ${ }^{3}$, que se sitúan en un mismo nivel de

3 Según la Real Academia Española, la definición de yuxtaposición es la siguiente: Del lat. iuxta 'junto a' y positǐo, -ōnis 'posición'. 1. f. Acción y efecto de yuxtaponer. 2. f. Geol. Modo de aumentar o crecer los minerales, a diferencia de los animales y vegetales, que crecen por intususcepción. 
jerarquía y se agrupan de tal manera que se hará hincapié en las relaciones que existan entre ellas. Esta clase de disciplinariedad presenta diversos elementos en un solo nivel, con numerosos objetivos para cada disciplina, independientes entre sí, pero con una línea de solidaridad y relación entendida por los fines que busca la línea jerárquica superior, sin una coordinación que permita su integración. En este sentido, Borrero (1989, citado en Miñana, 2000, p. 16) señala que se trata de una yuxtaposición, pero con predominancia de una disciplina en especial, lo cual permite reiterar la falta de cooperación y, en este caso, de toma de decisiones de dichas disciplinas.

La pluridisciplinariedad reúne disciplinas relativamente cercanas en el campo del conocimiento. Este proceso de interacción puede manifestarse en la comunicación de algunos sentires o ideas generales, de lo epistemológico, de los métodos comunes por utilizar, de los pasos para llevar a cabo un proceso, de la información sobre la cual se basaron para concluir y demás aspectos que los reúnen, sin necesidad de estar coordinados (Palmade, 1979).

La Organización para la Cooperación y el Desarrollo Económico (OCDE) realizó en 1970 el "Seminario Internacional sobre la Interdisciplinariedad en las Universidades", en el cual se recopilaron más de 25 experiencias en diferentes tipos de procesos disciplinarios. Entre los trabajos publicados, Apostel (1970) indica que la pluridisciplinariedad

es una yuxtaposición de disciplinas diversas situadas en el mismo nivel jerárquico y agrupadas subrayando sus relaciones. Sistema a un solo nivel y con objetivos múltiples; cooperación sin coordinación. (Apostel, 1970, p.145).

Se puede concluir que la pluridisciplinariedad podría relacionarse con lo que ocurre en las facultades de las universidades o en los distintos planes de estudio de una facultad, ya que, aunque convergen en un lugar, muy seguramente no están coordinadas bajo los mismos esquemas que conseguirán el logro de sus propósitos. 


\section{Aspectos institucionales}

Para identificar los aspectos disciplinarios dominantes en la estructura del programa de Contaduría Pública de la Universidad Santo Tomás, es necesario identificar los discursos dominantes que se evidencian en los documentos institucionales:

En primer lugar, el Proyecto Educativo Institucional-PEI (Universidad Santo Tomás, 2004b) indica en el "Análisis de los componentes de la misión" lo siguiente:

Los saberes generales interactúan con los especializados de manera orgánica o interdisciplinaria, pues estos se guían por la comprensión de la totalidad, y esta necesita alimentarse de las conclusiones científicas y de las consecuencias de su aplicación tecnológica. La USTA no es, pues, una yuxtaposición heterogénea de especialidades y unidades académicas con orientaciones independientes, sino un organismo académico-administrativo unificado, cuyos componentes convergen en una misión y visión compartidas. (Universidad Santo Tomás, 2004b, p. 20).

En el punto 1.3 del PEI (Perfil universitario y humanista de Tomás de Aquino), se enfatiza en las cualidades que, desde el pensamiento tomista, enmarcan la vida universitaria, conformando una personalidad científica. Una de dichas cualidades se fundamenta en la apertura interdisciplinaria y en la convergencia transdisciplinaria, que permiten pensar que el desarrollo de la actividad universitaria no es una actividad solitaria, ya que, si se piensa en el origen, será necesario determinar las cuestiones complejas con los docentes y estudiantes de todas las facultades.

Ahora bien, si se piensa en el desenlace de dicha actividad universitaria, deben considerarse las expectativas de los estudiantes, las cuales están enmarcadas en las necesidades de la región, en lo económico, político y social, lo cual requiere:

No solo el encuentro entre las disciplinas (filosofía, teología, derecho civil, derecho canónico, medicina), sino la indispensable construcción -mediante 
la búsqueda de nexos articuladores-de una visión que los trascendiese. (Universidad Santo Tomás, 2004 (a), p. 36).

Esto justifica la organización de facultades en unidades más amplias llamadas divisiones, que tienen una pretensión de organización interdisciplinaria, conectadas por unidades, como el Instituto Interdisciplinario de Investigaciones, la Vicerrectoría de Bienestar Universitario y el Centro de Comunicaciones. Precisamente, cuando la Universidad habla de currículo, sugiere la necesidad de incorporar nuevos elementos, articular lo disciplinario con las novedosas exigencias de interdisciplinariedad y transdisciplinariedad, que permiten acondicionarse a la vida nacional y mundial.

En este aspecto, el docente tiene un papel muy importante, ya que no proporcionará saberes definitivos, sino que deberá ayudar a desarrollar el intelecto desde las experiencias sensoriales en torno a problemas interdisciplinarios existentes.

Por otra parte, teniendo en cuenta la función investigativa, el PEI, indica que mediante la interdisciplinariedad, la universidad busca que las disciplinas tengan bases comunes, que puedan articular las investigaciones y en general los conocimientos, como exigencia clara de la complejidad actual. La investigación en la Universidad propiciará el análisis inter y trans de las disciplinas, sin excluir la investigación intra y multi. La transdisciplinariedad de los investigadores debe tener un discurso común que permita a todos vivir un lenguaje válido y respetable ante las demás ciencias, que hará:

... emerger de la confrontación de las disciplinas, en una indispensable etapa interdisciplinaria, nuevos datos articulatorios y una mirada unificadora necesaria para la comprensión holística. (Universidad Santo Tomás, 2002, p. 90).

Por su parte, en la Política de Investigación de la Universidad, se encuentra que la investigación: 
... implica un diseño metodológico que trabaja a partir de cuestionamientos, problemas y proyectos, relacionados directamente con líneas y con programas, aceptando la multicomplejidad como un referente inmediato.

... acepta el carácter inter y transdisciplinario (...) y requiere la configuración de los programas, acudiendo a las especificidades de las disciplinas, las facultades, los departamentos y los centros como puntos de partida del trabajo (Universidad Santo Tomás, 2009, p. 27).

En la investigación, entonces, según lo indicado en los documentos citados, todo lo relacionado con ella deberá tener un criterio interdisciplinario, que se centra en la complejidad de la ciencia, de los programas y de la academia en general.

Por su parte, la proyección social, como la intención final de las otras funciones sustantivas de la Universidad Santo Tomás, busca que la Institución se abra a la sociedad y ofrezca servicios articulados por las comunidades de docentes y estudiantes de cada unidad o de las comunidades interdisciplinarias de cada división.

Todo lo anterior permite identificar que en la documentación institucional de la universidad objeto de estudio, los conceptos de interdisciplinariedad no solo están descritos, sino que buscan, mediante el desarrollo de las funciones sustantivas, que sean interiorizados por toda la comunidad. Así pues, la Universidad Santo Tomás se basa en un concepto interdisciplinario que busca el desarrollo de su misión.

\section{Metodología}

El método de investigación es un aspecto importante y significativo en el ámbito del conocimiento científico. Según Cerda (1998), uno de los problemas más complejos que deben enfrentar los investigadores de esta época es la gran cantidad de métodos e instrumentos existentes que forman parte de posturas epistemológicas y escuelas filosóficas. 
El método es inherente a la metodología, que se puede observar desde varias perspectivas: a) como parte de la lógica que se ocupa del estudio de los métodos de investigación; y b) "la metodología entendida como el conjunto de aspectos operativos del proceso investigativo, y que es la concepción más conocida en el ambiente académico en general" (Bernal, 2010, p. 59). De acuerdo con ello, cuando se habla de investigación es normal referirse a un conjunto de elementos operativos que propician y permiten la realización del estudio.

El presente trabajo de investigación, se inscribe dentro de un método cualitativo que, de acuerdo con Bonilla y Rodríguez (2000, como se citó en Bernal, 2010), se va a orientar hacia la profundización de casos específicos y no hacia la generalización. Así pues, no se trata de medir, sino más bien de cualificar y posiblemente describir los fenómenos sociales de la forma como sean percibidos por los protagonistas que están en el interior de la investigación.

El enfoque cualitativo presume que los sujetos construyen su realidad de manera individual y distinguida, creando una subjetividad importante, consecuencia de la socialización en contextos particulares. En este sentido, Albert (2007), siguiendo a Guba y Lincoln (1985), Colás y Buendía (1992) y Anguera-Arnau-Ato (1998), considera unos principios acerca de la metodología cualitativa:

- Concepción múltiple de la realidad. Existen muchas realidades que no pueden ser consideradas de forma unitaria, por lo que cabe una diversificación de la interpretación de dicha realidad. Son diversas las facetas que se pueden estar considerando.

- El principal objetivo científico será la comprensión de los fenómenos. Se pretende llegar a captar las relaciones internas existentes indagando en la intencionalidad de las acciones, sin permanecer únicamente en la capa externa a la que parece propicia la descripción de los fenómenos.

- El investigador y el objeto de la investigación se interrelacionan de forma tal que se influyen mutuamente. 
- Se pretende un conocimiento de carácter ideográfico, de descripción de casos individuales. La investigación cualitativa no pretende llegar a abstracciones universales, y de ahí que abogue por el estudio de casos en profundidad, que luego se compararán con otros, con el fin de hallar regularidades y generar redes. Se pretende averiguar lo que es único y específico en un contexto determinado y lo que es generalizable a otras situaciones.

- La simultaneidad de los fenómenos e interacciones mutuas hace imposible distinguir las causas de los efectos.

- Los valores están implícitos en la investigación, reflejándose en las preferencias por un paradigma, elección de una teoría. (Albert, 2007, pp. 147-148).

Desde estos elementos, podría inferirse que el enfoque cualitativo busca la posibilidad de que los individuos, como parte de un grupo, expliquen la realidad vivida. Para Hernández, Fernández y Baptista (2010), en este enfoque existe una variedad de concepciones o modos de interpretar, ya que toda cultura o sistema de la sociedad tendrá una manera única de entender la realidad, y por ello admite subjetividad:

... puede concebirse como un conjunto de prácticas interpretativas que hacen el mundo "visible", lo transforman y convierten en una serie de representaciones en forma de observaciones, anotaciones, grabaciones y documentos. Es naturalista (porque estudia a los objetos... en sus contextos y ambientes naturales y cotidianidad) e interpretativo (pues intenta encontrar sentido a los fenómenos en función de los significados que las personas les otorgan). (Hernández, Fernández y Baptista, 2010, p. 10).

Así pues, en un enfoque cualitativo son variados los diseños de investigación que se pueden establecer. Hernández, Fernández y Baptista (2010) afirman que hay una gran variedad de tipologías de los diseños cualitativos. Lo anterior señala que en este tipo de método y de investigación, el diseño es abierto y flexible, y se puede desarrollar o construir a lo largo del trabajo. 
En síntesis, cabe resaltar que los resultados obtenidos de este trabajo no son desde ningún punto de vista generalizables a otros programas ni universidades.

\section{Estrategias para la recolección de datos}

La recolección de los datos estuvo orientada a obtener información que permitiese el entendimiento de los fenómenos indicados, por intermedio de las experiencias de los individuos y la revisión documental. El principal instrumento para este proceso es el investigador, y es apoyado por distintas técnicas que se pueden incorporar durante el proceso, como se indicó con anterioridad. Por ello, la manera para registrar los datos se va perfeccionando a medida que avanza la investigación.

Para el presente proyecto de investigación se utilizó, en primer lugar, un cuestionario y, en segunda instancia, una entrevista semiestructurada, lo cual permitirá contrastar y validar mutuamente la información que arroje el segundo instrumento. El cuestionario es el conjunto de preguntas diseñadas para conseguir los datos necesarios para alcanzar los objetivos de la investigación (Bernal, 2010).

La entrevista tiene una guía de preguntas y posteriormente los entrevistadores tienen la libertad de incorporar cuestionamientos adicionales que ayudarán a generar un espacio óptimo que facilite el perfeccionamiento del tema. Un apropiado clima de confianza y la empatía apropiada son fundamentales para lograr los objetivos (Esterberg, 2002).

\section{Diseño del cuestionario y la entrevista}

En la entrevista semiestructurada se genera un ambiente de conversación, donde el entrevistador-investigador no será un agente pasivo (escuchar), sino alguien que participa de manera activa, haciendo comentarios o novedosas preguntas que permitan al entrevistado ampliar sus ideas. En esta investigación, la indagación buscó identificar la interdisciplinariedad en 
el currículo del programa de Contaduría Pública de la Universidad Santo Tomás Bogotá, para luego ser contrastado con los documentos institucionales que indican su utilización.

Así pues, en este tipo de instrumento no se pueden establecer simples preguntas, sino focos que guían dichas preguntas. El foco principal de esta discusión en la interdisciplinariedad.

En las siguientes tablas se ilustran el cuestionario y las preguntas guía utilizadas u orientadoras realizadas con los protagonistas de la investigación:

Tabla 2. Formato de cuestionario inicial para evaluar la formalización de la interdisciplinariedad en el programa de Contaduría Pública de la Universidad Santo Tomás en Bogotá

Rol que desempeña:

Área:

Tiempo de servicio en el programa:

Por favor, califique las siguientes afirmaciones, marcando 1 cuando considere que es menos cierto y 5 cuando es más cierto.

\begin{tabular}{|c|c|c|c|c|c|c|}
\hline n. ${ }^{\circ}$ & AFIRMACIÓN & 1 & 2 & 3 & 4 & 5 \\
\hline 1 & $\begin{array}{l}\text { La interdisciplinariedad está formulada explícitamen- } \\
\text { te en el programa de Contaduría Pública. }\end{array}$ & & & & & \\
\hline 2 & $\begin{array}{l}\text { La intencionalidad de la interdisciplinariedad se } \\
\text { refleja claramente en la concepción del programa de } \\
\text { Contaduría Pública. }\end{array}$ & & & & & \\
\hline 3 & $\begin{array}{l}\text { Existen aspectos normativos relacionados con la pre- } \\
\text { sencia de la interdisciplinariedad en el currículo. }\end{array}$ & & & & & \\
\hline 4 & $\begin{array}{l}\text { La interdisciplinariedad del programa es evidenciable } \\
\text { en el escenario de aula. }\end{array}$ & & & & & \\
\hline 5 & $\begin{array}{l}\text { La interdisciplinariedad del programa es evidenciable } \\
\text { en el escenario del ejercicio profesional. }\end{array}$ & & & & & \\
\hline 6 & $\begin{array}{l}\text { La relación enseñanza-aprendizaje en el programa se } \\
\text { sustenta en la interdisciplinariedad. }\end{array}$ & & & & & \\
\hline
\end{tabular}




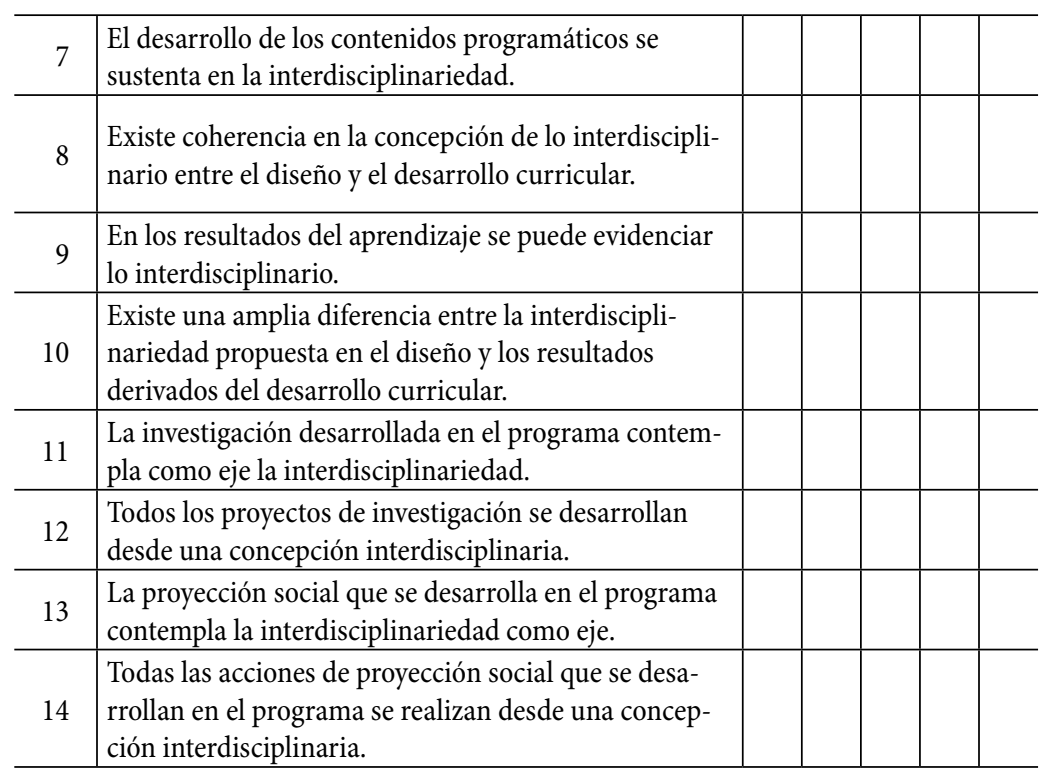

Fuente: Construcción propia. 
Tabla 3. Foco de conversación y preguntas orientadoras de la entrevista

\begin{tabular}{|c|c|}
\hline $\begin{array}{c}\text { Foco de } \\
\text { conversación }\end{array}$ & Preguntas orientadoras \\
\hline Interdisciplinariedad & $\begin{array}{l}\text { ¿Cómo se evidencia la interdisciplinariedad en el programa } \\
\text { de Contaduría Pública? } \\
\text { ¿Cómo se hace evidente la intencionalidad de la } \\
\text { interdisciplinariedad en la concepción del programa de } \\
\text { Contaduría Pública? } \\
\text { ¿Cómo se evidencian los aspectos normativos relacionados } \\
\text { con la presencia de la interdisciplinariedad en el currículo? } \\
\text { ¿Cómo se hace evidente la interdisciplinariedad del } \\
\text { programa en el escenario de aula? } \\
\text { ¿Cómo se hace evidente la interdisciplinariedad del } \\
\text { programa en el escenario del ejercicio profesional? } \\
\text { ¿Cómo se sustenta la interdisciplinariedad en la relación } \\
\text { enseñanza-aprendizaje en el programa? } \\
\text { ¿Cómo se evidencia la interdisciplinariedad en el desarrollo } \\
\text { de los contenidos programáticos? } \\
\text { ¿Cómo se evidencia que existe coherencia en la concepción } \\
\text { de lo interdisciplinario entre el diseño y el desarrollo } \\
\text { curricular? } \\
\text { ¿Cómo se evidencia lo interdisciplinario en los resultados } \\
\text { del aprendizaje? } \\
\text { ¿Existe una amplia diferencia entre la interdisciplinariedad } \\
\text { propuesta en el diseño y los resultados derivados del } \\
\text { desarrollo curricular? } \\
\text { ¿Cómo se evidencia que en la investigación desarrollada en } \\
\text { el programa se contempla como eje la interdisciplinariedad? } \\
\text { ¿Cómo se evidencia que todos los proyectos de investigación } \\
\text { se desarrollan desde una concepción interdisciplinaria? } \\
\text { ¿Cómo se hace evidente en la proyección social que se } \\
\text { desarrolla en el programa la interdisciplinariedad como eje? } \\
\text { ¿Cómo se evidencia que todas las acciones de proyección } \\
\text { social en el programa se realizan desde una concepción } \\
\text { interdisciplinaria? }\end{array}$ \\
\hline
\end{tabular}

Fuente: Construcción propia. 


\section{Protagonistas}

En el proceso cualitativo, la muestra corresponde a un grupo de personas, eventos, instituciones, entre otros, sobre el que se recolecta la información que ha de ser necesaria para el alcance de los objetivos, sin que represente toda la población del fenómeno social que se va a estudiar.

Creswell (2009, como se citó en Hernández, Fernández y Baptista, 2010, p. 394) indica que el muestreo cualitativo es de carácter propositivo. En este sentido, para elegir la muestra se basó en la capacidad operativa de recolección de información, el entendimiento del fenómeno y su naturaleza. En este caso se ha utilizado un tipo de muestra de expertos ${ }^{4}$ y se entrevistó a docentes que desarrollan procesos de las funciones sustantivas del programa de Contaduría Pública de la Universidad Santo Tomás, a saber: docencia-académico, investigación y proyección social.

\section{Análisis de resultados}

Para Hernández, Fernández y Baptista (2010), hay una gran diferencia entre la investigación cualitativa y la cuantitativa con relación al análisis de los datos:

En el proceso cuantitativo, primero se recolectan todos los datos y posteriormente se analizan, mientras que en la investigación cualitativa no es así (...) la recolección y el análisis ocurren prácticamente en paralelo; además, el análisis no es estándar, ya que cada estudio requiere de un esquema (...) propio de análisis. (Hernández, Fernández y Baptista, 2010, p. 439).

Por ello, se reciben datos de forma no estructurada que luego se estructuran. Para el análisis de la información, los investigadores utilizaron una matriz (tabla 4) con el fin de establecer los indicadores significativos y comunes, para posteriormente compararlos con los conceptos establecidos en

4 Tipo de muestra no probabilística, como lo señalan Hernández, Fernández y Baptista (2010). 
el apartado de "Aproximación conceptual" y así dar respuesta a la pregunta de investigación que dio origen al presente trabajo.

Tabla 4. Matriz para la realización del análisis comparado: aspectos significativos

\begin{tabular}{l|c|c|c|c}
\hline \multirow{2}{*}{ FOCO } & \multicolumn{4}{|c}{ Entrevistados } \\
\cline { 2 - 5 } & Ent.1 & Ent.2 & Ent.3 & Ent.n \\
\hline Interdisciplinariedad & & & & \\
\hline
\end{tabular}

Fuente: Construcción propia.

Posteriormente, se organizó, tabuló y analizó toda la información recogida por medio de los instrumentos indicados.

\section{Aspectos significativos}

Conforme a la tabla anterior, los principales aspectos significativos encontrados son los siguientes:

- La interdisciplinariedad como intención no se evidencia en los documentos del programa de Contaduría Pública de la Universidad Santo Tomás.

- La investigación en el programa sí refleja la interdisciplinariedad.

- En los espacios de aula no se refleja el ejercicio de la interdisciplinariedad.

- La interdisciplinariedad del programa es evidenciable en el ejercicio profesional.

- En la función proyección social no se evidencia la interdisciplinariedad.

- La concepción de lo interdisciplinario no se refleja en las acciones de proyección social.

- Existe coherencia entre la concepción de lo curricular y el diseño y desarrollo curricular. 
- Lo interdisciplinario se evidencia en los resultados del aprendizaje.

- La función sustantiva de docencia, sustentada en la relación enseñanzaaprendizaje, se sustenta en la interdisciplinariedad.

Derivado de lo expuesto atrás, se organizó, tabuló y analizó la información recogida mediante los instrumentos indicados, para posteriormente contrastarla con la documentación normativa institucional y de programa, y así poder concluir acerca del objetivo general de la investigación: identificar la presencia de la interdisciplinariedad en el currículo del programa de Contaduría Pública de la Universidad Santo Tomás Bogotá.

\section{Conclusiones}

Conforme a los diferentes desarrollos teórico-conceptuales acerca de la interdisciplinariedad y el currículo, y de acuerdo con lo encontrado en la relación de lo manifestado por los actores cuestionados y los documentos institucionales y de programa, se encuentra especialmente lo siguiente:

- Perfil del egresado de Contaduría Pública: "Cuenta con habilidades, destrezas y valores profesionales que le permiten trabajar en equipos disciplinarios e interdisciplinarios y generar valor en las organizaciones, proponiendo soluciones a situaciones problemáticas en diferentes contextos y promoviendo la confianza pública" (Universidad Santo Tomás, s. f.).

- Sin embargo, conforme a la revisión teórico-conceptual efectuada, se concluye que lo que se quiere señalar es trabajar en equipos disciplinarios y multidisciplinarios.

- El plan de estudios no es integrador, bien sea que se hable de su concepción o de su diseño.

- Lo que verdaderamente existe (sin mencionarlo explícitamente) es una concepción multidisciplinaria que se evidencia en diferentes espacios 
académicos, como en Derecho y Administración de Empresas. Sin embargo, como son ópticas independientes, no configura una visión de interdisciplinariedad dentro de la contaduría.

- En el ejercicio diario por parte de diferentes actores y documentos, existe una superposición de conceptos entre lo interdisciplinario y lo multidisciplinario, la cual dificulta incorporar en su verdadero sentido lo interdisciplinario.

- En el PEI se establece que desde 1974 las facultades se organizan en divisiones con intención "interdisciplinaria". Aunque reunir facultades en divisiones puede ayudar a instrumentalizar la interdisciplinariedad, no es menos cierto que esta, más que una concepción académicoadministrativa, es fundamentalmente epistemológica, que en términos ideales ha de derivar en una concepción y diseño curricular, y posteriormente en el microcurrículo (plan de estudios).

- Entonces, con relación a los objetivos específicos, se considera que se responden en la medida en que:

- Tanto en las respuestas como en los documentos institucionales se reconoce la existencia de la interdisciplinariedad dentro de los propósitos de formación del programa de Contaduría Pública de la Universidad Santo Tomás sede Bogotá, dado que todos lo hacen explícito, por lo menos en sus aspectos formales.

- Al pretender identificar la coherencia que existe entre la formulación explícita del programa y la interdisciplinariedad, esta no se refleja en términos conceptuales con exactitud sobre lo que es, sino que tiende a confundirse con otros términos, tales como la multidisciplinariedad.

- Esta investigación abre las puertas para futuros trabajos que deberán profundizar aspectos pedagógicos, didácticos, de enseñanzaaprendizaje, que permitan identificar las evidencias de lo planteado 
en el programa de Contaduría Pública de otras universidades, preferiblemente a nivel nacional, y desde las valoraciones de los docentes y los estudiantes.

\section{Referencias bibliográficas}

Agray Vargas, N. (2010). La construcción de currículo desde perspectivas críticas: una producción cultural. Signo y pensamiento, 29(56), 420-427. Recuperado de goo.gl/9PwKNx

Albert, M. (2007). La investigación educativa. Madrid: Mc Graw Hill.

Ander-Egg, E. (1994). Interdisciplinariedad en educación. Buenos Aires: Magisterio del Río de la Plata.

Anguera, M.T., Arnau, J., Ato, M.; Martínes, R.; Pascual, J. y Vallejo, G. (1998). Métodos de investigación en psicología. Madrid: Síntesis Psicología.

Apostel, L. (1970) -editor-. Interdisciplinarity : problems of teaching and research in Universities. Seminar on Interdisciplinarity In Universities Memories, University of Nice, France. OCDE Bernal, C. (2010). Metodología de la investigación: administración, economía, humanidades y ciencias sociales (3. ${ }^{\mathrm{a}} \mathrm{ed}$.). Bogotá: Pearson Educación.

Bolívar, A. (2008). Didáctica y currículum: de la Modernidad a la Postmodernidad. Archidona, Málaga: Editorial Aljibe.

Bonilla, E. y Rodríguez, P. (2000). Más allá del dilema de los métodos. La investigación en ciencias sociales. Bogotá: Universidad de los Andes, Grupo Editorial Norma.

Borrero, A. (1989). La interdisciplinariedad. Bogotá: Asociación Colombiana de Universidades, Fundación para la Educación Superior, ICFES.

Carvalho, J., Cadavid, L., Zapata, M., Tobón, F., Duque, M., Álvarez, M., Upegui, M., López, O., Machado, M., Ospina, C., Correa, J., Valencia, H. y Cano, A. (2006). Recreando el currículo: contaduría pública (Vol. Una propuesta del Comité de Apoyo Currículo.). Medellín, Colombia: Universidad de Antioquia.

Castro, S. (2013). Desafíos de la inter y la transdisciplinariedad para la Universidad en Colombia. Revista Trans-pasando fronteras, 3, 33-45. DOI: 10.18046/retf. i3.1625

Cerda, H. (1998). Los elementos de la investigación. Bogotá: El Búho. 
Colás, P. y Buendía, L. (1992). Investigación educativa. Sevilla: Alfar.

Creswell, J. W. (2009). Research Design: qualitative, quantitative, and mixed methods approaches (3. ${ }^{\mathrm{a}}$ ed.). Londres: Sage.

Díaz, A. (2003). Currículum. Tensiones conceptuales y prácticas. Revista Electrónica de Investigación Educativa, 5(2). Recuperado de goo.gl/IWnm3K

Duque, O. y Pinzón, J. (2014). Pensar el currículo: en la búsqueda de sentidos para la formación integral de contadores públicos. Lúmina, 15, 174-201. Recuperado de goo.gl/AtTx6G

Esterberg, K. (2002). Qualitative methods in social research. Nueva York: McGraw-Hill.

Gracia, E. (2002). Estado actual de la educación contable en Colombia. Ponencia presentada en el Segundo Foro Nacional sobre Educación Contable. Universidad de Cartagena, Cartagena. Recuperado de goo.gl/wHCnlU

Grajales, P. (2011). Formación interdisciplinar en el área de contabilidad gerencialy sistemas de información en la Pontificia Universidad Javeriana - Sede Bogotá: aproximaciones y propuestas (Tesis de grado). Pontificia Universidad Javeriana, Bogotá. Recuperada de goo.gl/M7qtVD

Hernández, R., Fernández, C. y Baptista, M. (2010). Metodología de la investigación (5.a ed.). México D. F.: McGraw-Hill Interamericana. Recuperado de goo.gl/Fa22TY

Kemmis, S. (1998). El currículo: más allá de la teoría de la reproducción (1. ${ }^{a}$ ed.) Madrid: Morata.

Lincoln, Y. S. y Guba, E. G. (1985). Naturalistic Inquiry. Beverly Hills: Sage.

Martín-Barbero, J. (2005). Transdisciplinariedad: notas para un mapa de sus encrucijadas cognitivas y sus conflictos culturales. En J. E. Jaramillo (Comp.), Cultura, identidades y saberes fronterizos. Bogotá: Universidad Nacional de Colombia.

Bolivar, O; Martínez, G; Gracia, E . (2002). El rediseño curricular contable. Entre lo profesional y lo disciplinar. En C. C. C-Cinco, Del hacer al saber. Realidades y Perspectivas de la educación contable en Colombia (pp. 113-160). Popayán: Universidad del Cauca.

Menéndez, F. (1998). Interdisciplinariedad y multidisciplinariedad en salud mental. Revista de la Asociación Especialistas de Neuropsiquiatría, 18(65), 145-150. Recuperado de goo.gl/R0nPHW 
Miñana, C. (2000). Interdisciplinariedad y currículo. En. C. Miñana (Ed.), Interdisciplinariedad y currículo. Construcción de proyectos de escuela-universidad. Memorias del V Seminario Internacional (pp. 1-74). Bogotá: Universidad Nacional de Colombia. Recuperado de goo.gl/bJpU4F

Morin, E. (2001). De la reforma universitaria. UNI-PLURI/DIVERSIDAD, 1(2). Recuperado de goo.gl/DtFhWd . (2010). Sobre la interdisciplinariedad. Orientaciones Universitarias. La Interdisciplinariedad en la Universidad, 43, 9-17. Recuperado de goo.gl/4xhRus Nicolescu, B. (1996). La transdisciplinarité. Manifeste. París: Le Rocher

Ortiz, E. (12 de 2006). Retos y perspectivas del currículum integrado. Cuaderno de Investigación en la Educación, 21, 35-56. Recuperado de goo.gl/nHxjdM

Palmade, G. (1979). Interdisciplinariedad e ideologías. Madrid: Narcea de Ediciones. Popkewitz. T. (2007). Historia del curriculum: una anotación breve de la historia. Profesorado. Revista de Curriculum y Formación de Profesorado, 11(3), 1-3. Recuperado de goo.gl/160ApW

Real Academia Española. (2014). Diccionario de la lengua española. Madrid: Espasa. Roldán, M. (2008). Bioética y enfermería. Diálogo transdisciplinario. Recuperado de Recuperado de goo.gl/ugaJUw

Stake, R. (2006). Evaluación comprensiva. Barcelona: Graó.

Stenhouse, L. (2003). Investigación y desarrollo del currículum. Madrid: Morata.

Taba, H. (1974). Elaboración del currículo (Trad. R. Albert). Buenos Aires: Editorial Troquel S. A. Recuperado de goo.gl/ht045j

Tamayo y Tamayo, M. (2011). La interdisciplinariedad. Santiago de Cali: Universidad ICESI.

Torres, J. (2006). Globalización e interdisciplinariedad: el curriculum integrado. Madrid: Ediciones Morata.

Universidad Santo Tomás (2002). Perfil del egresado. Recuperado de http://contaduria.usta.edu.co/index.php/presentacion-contaduria-publica/perfil-egresado .(2004a). Política curricular para programas académicos. Bogotá: USTA. . (2004b). Proyecto Educativo Institucional (PEI). Bogotá: USTA. . (2009). Política de investigación en la Universidad Santo Tomás. Bogotá: USTA.

Zabalza, M. A. (1801). Diseño y desarrollo curricular. Madrid: Narcea. 